\title{
Applying DevOps to Machine Learning Processes: A Systematic Mapping
}

\author{
Beatriz Mayumi Andrade Matsui ${ }^{1}$, Denise Hideko Goya ${ }^{1}$ \\ ${ }^{1}$ Centro de Matemática, Computação e Cognição, Universidade Federal do ABC \\ Av. dos Estados, 5001 - Bairro S. Terezinha, Santo André, SP, Brazil - CEP: 09210-580 \\ \{beatriz.mayumi, denise.goya\}@ufabc.edu.br
}

\begin{abstract}
DevOps practices have been increasingly used by software engineering teams in order to improve development stages. In processes that involve machine learning (ML), DevOps can also be applied to deploy machine learning models into production - a practice also known as MLOps. This systematic mapping aims to understand how DevOps has been applied to machine learning processes and what challenges are faced. Most of the 15 articles selected make use of CI/CD practices and propose architectures for the implementation of $M L$ models. The biggest challenges are the characteristics inherent to the ML models and resistance to change.
\end{abstract}

Resumo. Práticas de DevOps têm sido cada vez mais utilizadas por equipes de engenharia de software com o intuito de aprimorar as etapas de desenvolvimento. Em processos que envolvem machine learning (ML), DevOps também pode ser aplicado a fim de implantar modelos de aprendizado de máquina em produção - prática também conhecida como MLOps. Neste mapeamento sistemático objetiva-se entender como DevOps tem sido aplicado a processos de machine learning e quais são os desafios enfrentados. Foram selecionados 15 artigos e observou-se que a maioria faz uso de práticas de CI/CD e propõe arquiteturas para a implantação de modelos de ML. Como maiores desafios, têm-se as características inerentes aos modelos de ML e resistência à mudança.

\section{Introdução}

Práticas de DevOps têm sido cada vez mais utilizadas por equipes de desenvolvimento de software, com o intuito de automatizar e simplificar processos que vão desde a integração, passando por etapas de teste, homologação, implantação até a entrega final da aplicação aos usuários, em um cenário de constantes atualizações de versões de código [Leite et al. 2019].

O termo "DevOps" representa a combinação de Desenvolvimento e Operações, e pode ser entendido como uma cultura de colaboração, "uma nova forma de pensar" no âmbito da engenharia de software [Jabbari et al. 2016]. Seus principais objetivos consistem em remover as lacunas existentes entre as áreas de desenvolvimento e operações e, com isso, garantir que o desenvolvimento, implantação e operação de aplicativos e sistemas ocorram com alta qualidade [Agrawal and Rawat 2019]. Da mesma forma, machine learning (ML), ou aprendizado de máquina, é um campo de estudo cada vez mais em ascensão, cujas técnicas são utilizadas em diferentes áreas da indústria, tais como: saúde, 
educação e negócios, a fim de automatizar processos e permitir que atividades humanas sejam otimizadas e concluídas com mais velocidade e precisão [Langley and Simon 1995].

A construção de um modelo de aprendizado de máquina envolve algumas etapas características, que vão desde a coleta e preparação dos dados, até o treinamento, validação e teste [Domingos 2012]. Equipes que trabalham com aprendizado de máquina têm suas próprias metodologias e abordagens de trabalho, sobretudo aquelas relacionadas à ciência de dados [Saltz et al. 2017]. Muitas dessas equipes ainda não estão acostumadas a práticas de DevOps, as quais poderiam ser muito úteis para o desenvolvimento, implantação e monitoramento [Schlossnagle 2018] dessas aplicações, em um ciclo contínuo de melhoria do sistema.

Alguns dos maiores desafios relacionados à adoção de práticas de DevOps por times de engenharia de software (incluindo engenheiros, pesquisadores e demais envolvidos no processo), se referem sobretudo a: i) complexidade de implementar pipelines de entrega contínua para sistemas originalmente monolíticos, e os desafios de redesenhá-los para uma estrutura de microsserviços [Shahin et al. 2016]; ii) como a organização deve ser reestruturada para adotar DevOps - questão ainda não respondida totalmente pela literatura; iii) como avaliar a qualidade das práticas de DevOps que têm sido implementadas nas organizações - para isso, no estudo de [Forsgren and Kersten 2018] sugere-se a coleta de dados de pesquisa (survey) e do sistema para mensurar as transfomações trazidas com DevOps, e os pesquisadores de [de Feijter et al. 2018] propõem um modelo de maturidade de DevOps para ajudar as organizações a medirem seus níveis atuais e identificarem quais áreas precisam de mais investimento. Contudo, o fato é que nenhum desses modelos ainda é adotado amplamente na indústria [Forsgren and Kersten 2018], tampouco encontram-se muito representados na literatura.

Também para os times que trabalham com aprendizado de máquina, muitos são os desafios inerentes ao desenvolvimento e à melhoria contínua desses sistemas, sobretudo no que concerne à coleta, extração e limpeza dos dados [Karamitsos et al. 2020]. A dívida técnica (technical debt) [Tom et al. 2013, Sculley et al. 2015], por exemplo, é apontada no estudo de [Sculley et al. 2015] como um dos principais problemas ocasionados com o desenvolvimento de modelos de machine learning, devido a dificuldades relacionadas à manutenção do código e outras características inerentes aos mesmos. No entanto, poucos ainda são os estudos que abordam essa temática ou propõem frameworks de DevOps para solucionar tais problemas.

Com isso, muitos questionamentos concernentes à aplicação de DevOps em processos de machine learning e melhores práticas ainda se encontram sem respostas claras presentes na literatura, fato que o mapeamento sistemático aqui apresentado visa analisar e compreender. Desta forma, objetiva-se traçar um panorama inicial sobre a área, investigar em que estado se encontram as atuais pesquisas e servir como fonte de referência para futuros pesquisadores do tema, especialmente no Brasil.

\section{Metodologia}

O método seguido é o de mapeamento sistemático da literatura, que consiste na investigação e análise de um tema, com o objetivo de responder a questões de pesquisa que guiarão o processo de busca [Petersen et al.. 2008]. É um estudo secundário, que visa 
obter uma visão ampla do estado atual de determinada área [Moher et al. 2015], sendo realizado geralmente quando da escassa disponibilidade de estudos na literatura sobre o tema alvo [Kitchenham and Charters 2007].

No presente trabalho, o objetivo é investigar como DevOps tem sido aplicado a práticas de machine learning (processo também conhecido como "MLOps", do inglês Machine Learning + Operations), de forma que seja possível descobrir como isso está contribuindo para otimizar processos e ajudar profissionais que fazem uso dessas práticas no desenvolvimento de seus sistemas.

Para tal fim, é necessária a definição de questões de pesquisa (2.1), string de busca (2.2), critérios de inclusão e exclusão (2.3), bases de busca (2.4), seleção dos artigos relevantes para o mapeamento (2.5), extração e análise dos resultados (3), passos esses descritos nas subseções a seguir.

\subsection{Questões de pesquisa}

Neste trabalho são definidas as seguintes questões de pesquisa (QP):

- QP1: Como DevOps tem sido aplicado na prática em processos de machine learning?

- QP2: Quais são os atuais desafios da implementação de DevOps no contexto de machine learning (MLOps)?

A QP1 visa descobrir em quais cenários DevOps tem sido aplicado a práticas de machine learning, e de que forma isso está sendo realizado e reportado na literatura. A QP2 tem como objetivo investigar quais são os desafios mais comumente enfrentados por aqueles que estão inserindo práticas de DevOps em seus processos de ML.

Essas perguntas guiarão o processo de busca dos artigos e a partir delas será extraída a string de busca, como pode ser visto na subseção 2.2 a seguir.

\subsection{String de busca}

Strings de busca são sequências de caracteres que contemplam os termos-chave do trabalho, a partir das questões de pesquisa [Kitchenham et al. 2009]. A string de busca definida nesse estudo contempla os seguintes termos: devops, "machine learning", "aprendizado de máquina" e mlops, como pode ser visto na Tabela 1.

Tabela 1. String de busca definida para o mapeamento sistemático

\begin{tabular}{|l|l|}
\hline String de busca & (devops AND ("machine learning" OR "aprendizado de máquina")) OR mlops \\
\hline
\end{tabular}

\subsection{Critérios de inclusão e exclusão}

Como critérios de inclusão para a seleção dos trabalhos, foram definidos:

- CI1: artigo científico publicado em revista ou periódico.

- CI2: publicação entre 2017 e julho de 2021.

E como critérios de exclusão, definiu-se:

- CE1: estudo não se refere à aplicação de DevOps com machine learning. 
- CE2: texto completo não está disponível.

- CE3: não está em inglês ou português.

- CE4: artigos repetidos.

- CE5: estudos secundários.

Além disso, definiu-se como critério de qualidade que o artigo deveria ser completo e possuir no mínimo 5 páginas, pois caso contrário não seria incluído no mapeamento. Um critério de qualidade foi a inclusão exclusiva de artigos de periódicos científicos, com a intenção de priorizar pesquisas em estágio mais avançado de experimentação, avaliação ou análise.

\subsection{Bases de busca}

Para a seleção dos artigos foram utilizadas as seguintes bases de busca:

- Web of Science

- IEEE Xplore

- ScienceDirect

- ACM Digital Library

- SpringerLink

A escolha dessas bases levou em consideração a relevância das mesmas no âmbito da ciência da computação e pesquisa, e a possibilidade de encontrar mais estudos sobre o tema alvo do mapeamento sistemático. O acesso às bases se deu por meio de acesso institucional federado via Portal de Periódicos CAPES.

\subsection{Seleção dos artigos}

Para a seleção dos artigos, foram aplicados os critérios de inclusão e exclusão de acordo com protocolo definido anteriormente e apresentado na subseção 2.3 desse artigo. A relação da quantidade de estudos selecionados ao aplicar os critérios de inclusão (CI1 e CI2) e critérios de exclusão (CE1 a CE5) por base de pesquisa pode ser vista na Tabela 2.

Tabela 2. Quantidade de artigos selecionados por base de busca em cada etapa

\begin{tabular}{|c|c|c|c|}
\hline Base de busca & CI1, CI2 & CE1, CE2, CE3, CE4, CE5 & Total \\
\hline Web of Science & 14 & 5 & 5 \\
\hline IEEE Xplore & 7 & 2 & 2 \\
\hline ScienceDirect & 8 & 2 & 2 \\
\hline ACM Digital Library & 20 & 1 & 1 \\
\hline SpringerLink & 127 & 5 & 5 \\
\hline
\end{tabular}

Ao total, foram selecionados 15 artigos. Na Figura 1 é possível visualizar a distribuição da quantidade de artigos por ano de publicação. 


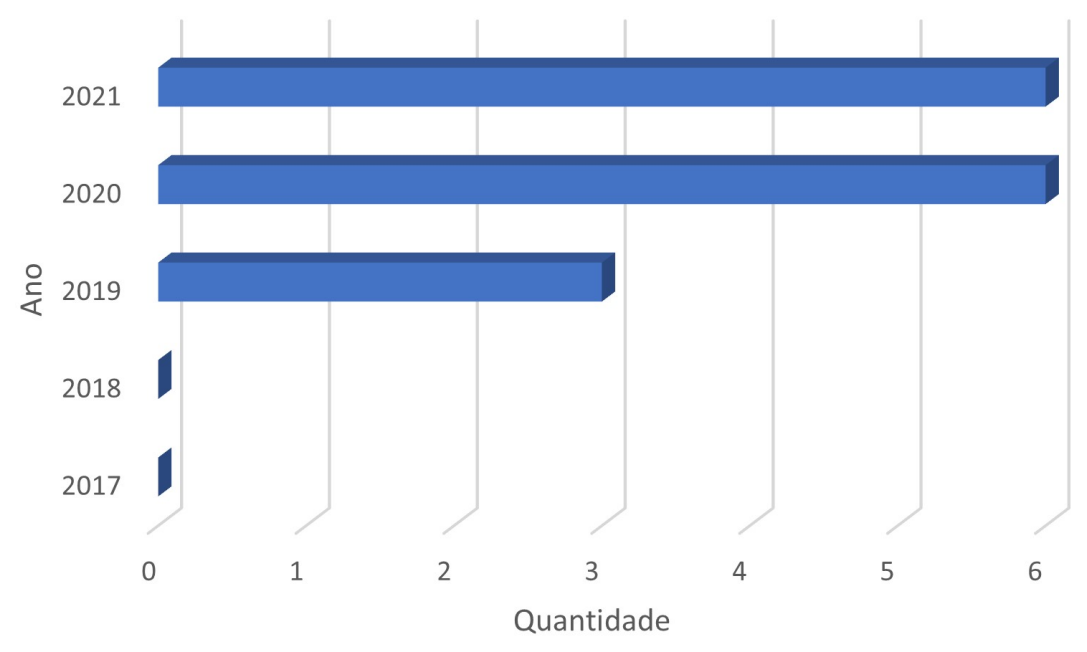

Figura 1. Relação da quantidade de artigos selecionados por ano

Nota-se que a maior quantidade de estudos data dos anos de 2020 e 2021, sendo o número total somente dos últimos três anos (2019-2021).

\section{Resultados e Discussão}

Com a análise dos 15 artigos selecionados foi possível obter respostas às questões de pesquisa definidas e, assim, traçar um panorama inicial sobre a aplicação de DevOps em processos de machine learning - como tem sido realizada e retratada na literatura atualmente. Nas subseções 3.1 e 3.2 a seguir são descritos os resultados obtidos para as questões de pesquisa QP1 e QP2, respectivamente. Na Tabela 3 é possível visualizar a relação de trabalhos selecionados, com o mapeamento dos artigos (listados de A1 a A15) às suas respectivas referências.

\subsection{Respostas à $Q P 1$}

- QP1: Como DevOps tem sido aplicado na prática em processos de machine learning?

Cerca de 53\% dos artigos mencionam práticas de CI/CD (Integração Contínua e Implantação/Entrega Contínua ou, do inglês, Continuous Integration e Continuous Deployment/Delivery) na implantação de modelos de machine learning. São eles: A3, A4, A7, A8, A9, A11, A12 e A15.

Em A3, de [Castellanos et al. 2021], é proposto um modelo de arquitetura para análise de big data que, em sua implementação, faz uso das práticas DevOps de Infraestrutura como Código (Infrastructure as Code, $I a C$ ), conteinerização e arquitetura serverless (sem servidor). Em A4, [Dalla Palma et al. 2021] detalham o uso de práticas de machine learning e DevOps para identificar e prever scripts de IaC com defeitos, servindo como referência para profissionais e pesquisadores de DevOps, como o próprio estudo indica. A7, de [García et al. 2020], foca nas práticas de CI/CD, relatando como alterações no código-fonte são automaticamente validadas com CI, e como a nova versão do modelo é disponibilizada também de forma automática por meio de CD. Em A8, os autores [Granlund et al. 2021] descrevem conceitualmente práticas de entrega contínua em software e 
o ciclo de vida de MLOps; também apresentam um estudo de caso sobre uma aplicação médica voltada para a avaliação de riscos de cirurgia de substituição de articulações, que poderia se beneficiar das práticas de MLOps em seu desenvolvimento (incluindo requisitos e implicações regulatórias). O artigo A9, de [Karamitsos et al. 2020], apresenta práticas, princípios e ferramentas de CI/CD para o desenvolvimento e implantação de modelos de ML, com o objetivo de suportar ciclos de feedback rápidos, descobrir dívida técnica oculta, aperfeiçoar funções operacionais e a entrega de valor. Em, A11, [Li et al. 2020] dão enfoque a práticas de "AIOps" (Artificial Intelligence for IT Operations) para prever falhas de nó em aplicações de grande escala na nuvem. O estudo A12, de [Liu et al. 2020], introduz o ciclo de vida de MLOps e investiga processos e ferramentas de CI/CD para implantação de modelos de ML, propondo a adoção de frameworks de código aberto para esse fim. Por fim, A15, dos autores [Spjuth et al. 2021], apresentam uma discussão em torno da aplicação de aprendizado de máquina na descoberta de novos medicamentos, e como MLOps pode contribuir para a criação e implementação de um modelo robusto de ML que atenda a esse cenário.

Observou-se também que $60 \%$ do total de artigos selecionados propõem frameworks ou arquiteturas que envolvem o aperfeiçoamento de práticas de ML, para diferentes fins, por meio da utilização de conceitos e práticas de DevOps. São eles os estudos A1, A2, A3, A4, A6, A7, A10, A12 e A14.

No artigo A1, de [Barisits et al. 2019], é apresentado um modelo de software de código aberto para gestão de dados de pesquisas científicas, com o uso de metodologias ágeis e DevOps. Em A2, [Bersani et al. 2019] propõem um framework para detectar antipadrões em arquiteturas de big data com a utilização de pipelines de DevOps. Em A3, que também menciona práticas de CI/CD, [Castellanos et al. 2021] apresentam um modelo específico de domínio juntamente a práticas de DevOps a fim de criar, implantar e monitorar métricas de desempenho em aplicações de análise de big data. No estudo A4, de [Dalla Palma et al. 2021], é proposto um framework para a detecção de defeitos em scripts de IaC. Os autores desse estudo apontam que a previsão eficaz de scripts de $\mathrm{IaC}$ propensos a falhas permite que metodologias de DevOps sejam aplicadas com enfoque nessa área, auxiliando na garantia de qualidade. Em A6, [Fursin 2021] propõe um framework para a organização de projetos de pesquisa como um banco de dados de componentes reutilizáveis e fluxo de dados automatizado. O artigo A7, de [García et al. 2020], apresenta uma arquitetura distribuída para prover àqueles que trabalham com aprendizado de máquina ferramentas e serviços de nuvem para a melhoria do processo de implantação dos modelos. A10, dos autores [Karn et al. 2019], apresenta uma metodologia de nuvem e arquitetura DevOps para seleção e ajuste automáticos de parâmetros em modelos de ML, tendo como base a orquestração de contêineres. Em A12, de [Liu et al. 2020], é apresentada uma aplicação construída a partir de código aberto para validar MLOps no contexto de detecção de vulnerabilidade em código. Já em A14, dos autores [Pääkkönen and Pakkala 2020], propõe-se uma arquitetura de referência para sistemas de big data com o uso de machine learning e DevOps.

Os demais artigos (A5 e A13) não propõem especificamente um framework ou uma solução inovadora relacionada a práticas de MLOps - são mais teóricos e voltados à indústria - mencionam a utilização de DevOps em cenários tais como o de IoT (Internet of Things, ou "Internet das Coisas"), envolvendo tecnologia analítica de processos e sensores 
inteligentes [Eifert et al. 2020], e análise de aplicação de práticas MLOps na construção de pipelines para planejamento e controle da produção [Oluyisola et al. 2021].

$\mathrm{Na}$ Tabela 3 visualiza-se uma síntese dos resultados aqui descritos, com a relação dos artigos e se os mesmos mencionam práticas de CI/CD, propõem frameworks e qual a área de enfoque da aplicação do estudo. Na coluna Implementação, o "Sim" indica que o artigo, além de discutir conceitos, também descreveu resultados da implementação que se propôs a fazer; a resposta "Não" referencia o artigo que apresentou discussões mais teóricas sem propor uma arquitetura ou implementação.

Tabela 3. Relação dos artigos e tópicos abordados

\begin{tabular}{|c|c|c|c|c|c|}
\hline Artigo & Referência & Implementação & CI/CD & Framework & Área de aplicação \\
\hline A1 & $\begin{array}{l}\text { [Barisits et al. } \\
\text { 2019] }\end{array}$ & Sim & Não & Sim & Gestão de dados \\
\hline$\overline{\mathrm{A2}}$ & $\begin{array}{l}\text { [Bersani et al. } \\
\text { 2019] }\end{array}$ & Sim & Não & Sim & Análise de big data \\
\hline A3 & $\begin{array}{l}\text { [Castellanos } \\
\text { et al. 2021] }\end{array}$ & Sim & Sim & Sim & Análise de big data \\
\hline A4 & $\begin{array}{l}\text { [Dalla Palma } \\
\text { et al. 2021] }\end{array}$ & Sim & Sim & Sim & $\begin{array}{l}\text { Predição de defeitos; } \\
\mathrm{IaC}\end{array}$ \\
\hline A5 & $\begin{array}{l}\text { [Eifert et al. } \\
\text { 2020] }\end{array}$ & Não & Não & Não & $\begin{array}{l}\text { Sensores inteligen- } \\
\text { tes; IoT }\end{array}$ \\
\hline A6 & [Fursin 2021] & Sim & Não & Sim & $\begin{array}{l}\text { Gestão de dados; } \\
\text { automação }\end{array}$ \\
\hline A7 & $\begin{array}{l}\text { [García et al. } \\
\text { 2020] }\end{array}$ & Sim & Sim & Sim & $\begin{array}{l}\text { Computação em nu- } \\
\text { vem; aprendizado de } \\
\text { máquina }\end{array}$ \\
\hline A8 & $\begin{array}{l}\text { [Granlund et al. } \\
\text { 2021] }\end{array}$ & Sim & Sim & Não & $\begin{array}{l}\text { Gestão de dados; } \\
\text { aprendizado de má- } \\
\text { quina }\end{array}$ \\
\hline A9 & $\begin{array}{l}\text { [Karamitsos } \\
\text { et al. 2020] }\end{array}$ & Sim & Sim & Não & $\begin{array}{l}\text { Aprendizado de má- } \\
\text { quina }\end{array}$ \\
\hline A10 & $\begin{array}{lll}\text { KKarn } & \text { et } & \text { al. } \\
\text { 2019] } & & \end{array}$ & Sim & Não & Sim & $\begin{array}{l}\text { Computação em nu- } \\
\text { vem; aprendizado de } \\
\text { máquina }\end{array}$ \\
\hline A11 & [Li et al. 2020] & Sim & Sim & Não & $\begin{array}{l}\text { Computação em nu- } \\
\text { vem; aprendizado de } \\
\text { máquina }\end{array}$ \\
\hline A12 & [Liu et al. 2020] & Sim & Sim & Sim & $\begin{array}{l}\text { Aprendizado de má- } \\
\text { quina }\end{array}$ \\
\hline A13 & $\begin{array}{l}\text { [Oluyisola et al. } \\
\text { 2021] }\end{array}$ & Não & Não & Não & $\begin{array}{l}\text { Planejamento e con- } \\
\text { trole da produção }\end{array}$ \\
\hline A14 & $\begin{array}{l}\text { [Pääkkönen and } \\
\text { Pakkala 2020] }\end{array}$ & Sim & Não & Sim & Análise de big data \\
\hline A15 & $\begin{array}{l}\text { [Spjuth et al. } \\
\text { 2021] }\end{array}$ & Não & Sim & Não & Gestão de dados \\
\hline
\end{tabular}

Pode-se observar que boa parte dos estudos selecionados faz uso de práticas de 
CI/CD. A Integração Contínua (CI) é uma prática de DevOps que consiste em ter a fase de desenvolvimento do software totalmente automatizada, com a validação do código acontecendo assim que as alterações são confirmadas e mescladas em um repositório central no sistema de controle de versão de origem [Rossel 2017]. A Entrega Contínua (CD), por sua vez, refere-se à fase que vem logo depois: a entrega automatizada da aplicação. Quando há um novo artefato de construção, uma liberação é acionada e o artefato é implementado automaticamente no ambiente desejado.

O que é possível perceber com os resultados encontrados é que já existem exemplos disponíveis na literatura da aplicação dessas práticas de CI/CD em diferentes contextos que envolvem machine learning. Os processos de implantação de modelos de ML são essencialmente baseados na iteração - o que pode levar horas ou até dias, dependendo do tamanho do modelo, até que o mesmo seja totalmente treinado e testado. As pesquisas apontam que DevOps pode sim auxiliar na estabilização e otimização desses processos, por meio de CI/CD e outras abordagens ágeis.

Percebe-se também que enquanto alguns estudos possuem um objetivo específico de implementação (como A1, A2 e A15), outros são mais genéricos e abordam a temática de MLOps de forma mais ampla, aplicável a diversos cenários que envolvam machine learning, a exemplo dos estudos A7 e A9.

\subsection{Respostas à $\mathrm{QP2}$}

- QP2: Quais são os atuais desafios da implementação de DevOps no contexto de machine learning (MLOps)?

A maioria dos desafios mencionados em relação à adoção de DevOps no contexto de machine learning se refere sobretudo às próprias características inerentes ao sistemas e modelos de $\mathrm{ML}$ - os quais, diferentemente dos sistemas tradicionais de engenharia de software, possuem a necessidade de serem constantemente treinados e testados, em um processo de iteração contínuo [Lwakatare et al. 2020]. Tais desafios são mencionados no estudo A8, que aborda a questão das etapas necessárias para implantar modelos de machine learning, considerando treino, teste e validação, e a importância do monitoramento, que deve considerar vieses e desvios que podem surgir ao longo do processo de treinamento [Granlund et al. 2021].

Também destacam-se como desafios comuns: a resistência à mudança; times que trabalham isolados, em "silos"; ausência de profissionais capacitados; pesquisas ainda incipientes na área. No artigo A9, de [Karamitsos et al. 2020], a mudança de cultura é apontada como um dos principais desafios no processo de adoção de práticas de DevOps para ML, bem como o trabalho isolado e a falta de entendimento do valor que tais práticas podem agregar ao negócio. [Granlund et al. 2021] em A8 também ressaltam as dificuldades relacionadas à integração dos agentes envolvidos no contexto de ML, que geralmente possuem atribuiçõos separadas (por exemplo: desenvolvedores responsáveis somente pelo código e liberação do sistema para produção; engenheiros e cientistas de dados dedicados exclusivamente à coleta de dados e geração do modelo), o que leva à desarmonia dos processos, atrasos na entrega e conflitos.

Os pesquisadores de A14, em [Pääkkönen and Pakkala 2020], citam que a adoção de DevOps em práticas de machine learning traz novos desafios, em comparação a DevOps aplicado somente ao contexto da engenharia de software. Dentre eles, destacam-se 
a eventual baixa qualidade dos dados para modelagem, uma possível escassez de dados rotulados e a necessidade de melhoria e treinamento contínuos, fatores tais que tornariam mais difícil a implementação de DevOps no contexto de ML.

No artigo A11, [Li et al. 2020] mencionam os desafios inerentes a predições com grandes quantidades de dados para monitoramento, e a complexidade da análise dos sintomas de falha. [Spjuth et al. 2021] em A15, por sua vez, destacam as características do ciclo de vida de ML, a dívida técnica [Sculley et al. 2015], além de desafios relacionados a monitoramento e governança.

Percebe-se com isso que os principais desafios da adoção de DevOps por aqueles envolvidos em processos de ML, de acordo com as pesquisas selecionadas, são realmente as próprias caraterísticas dos modelos e ciclo de vida de ML, a mudança de mentalidade e cultura nas organizações e nas diversas equipes envolvidas e, também, o desconhecimento ou muitas vezes falta de acesso a ferramentas e melhores práticas - sobretudo considerando que as pesquisas na área ainda são muito recentes (vide os estudos aqui selecionados que datam apenas de 2019 a 2021, mesmo com o critério de inclusão considerando artigos a partir de 2017).

\section{Considerações Finais}

O presente mapeamento sistemático realizou o levantamento da literatura relacionada ao tema de DevOps aplicado a processos de machine learning, a partir de duas questõeschave: "Como DevOps tem sido aplicado em processos de machine learning?", e "Quais os atuais desafios da implementação de DevOps no contexto de ML?". Definida a string de busca e aplicados os critérios de inclusão e exclusão, foram selecionados 15 artigos para análise.

Observou-se que a maioria dos estudos menciona a utilização de práticas de integração e entrega contínuas (CI/CD) para a implantação de modelos de ML, e/ou apresentam arquiteturas ou frameworks para melhor abordar esses cenários. Como atuais desafios, destacaram-se as características inerentes aos modelos de ML, que tornam mais difícil operá-los continuamente em produção, a dificuldade de integração dos times e a resistência a mudanças.

Nota-se a ausência de estudos em língua portuguesa, o que gera um interessante ponto para reflexão sobre os locais de onde estão vindo as pesquisas, assim como o público-alvo (se regional ou global, etc.). Dentre as autorias dos artigos selecionados nesse mapeamento sistemático, por exemplo, a maior contribuição vem de países como Estados Unidos, Canadá, Itália e Alemanha, seguidos por outros países da Europa, refletindo uma maior quantidade de pesquisas em andamento ou finalizadas nesses países mais desenvolvidos.

É importante citar que o protocolo adotado para realizar o mapeamento impõe algumas restrições, tais como i) a escolha das bases que pode não ter alcançado toda a diversidade de trabalhos relacionados ao tema; ii) a inclusão exclusiva de artigos de periódicos científicos que pode ter eliminado trabalhos apresentados em conferências; iii) a string de busca que poderia levar em consideração outros termos; e iv) possíveis erros humanos na seleção dos artigos.

Como trabalhos futuros, pode-se pensar na investigação da produção científica so- 
bre o tema aqui apresentado no Brasil - quais são e como têm sido adotadas as práticas de DevOps no contexto de ML por nossas empresas, indústrias e demais instituições? Além disso, sugere-se que mais pesquisas sejam realizadas considerando sobretudo o ambiente acadêmico - vê-se que esse é um tema mais abordado por empresas envolvidas com desenvolvimento e machine learning, pela natureza prática do mesmo - mas podem haver também benefícios em se investigar tal temática no ambiente das universidades e instituições de pesquisa, sobretudo no Brasil, uma vez que DevOps e ML podem contribuir para o desenvolvimento de diferentes áreas - da academia à indústria - no país.

\section{Referências}

Agrawal, P. and Rawat, N. (2019). Devops, a new approach to cloud development \& testing. In 2019 International Conference on Issues and Challenges in Intelligent Computing Techniques (ICICT), volume 1, pages 1-4. IEEE.

Barisits, M., Beermann, T., Berghaus, F., Bockelman, B., Bogado, J., Cameron, D., Christidis, D., Ciangottini, D., Dimitrov, G., Elsing, M., et al. (2019). Rucio: Scientific data management. Computing and Software for Big Science, 3(1):1-19.

Bersani, M. M., Marconi, F., Tamburri, D. A., Nodari, A., and Jamshidi, P. (2019). Verifying big data topologies by-design: a semi-automated approach. Journal of Big Data, 6(1):1-23.

Castellanos, C., Varela, C. A., and Correal, D. (2021). Accordant: A domain specificmodel and devops approach for big data analytics architectures. Journal of Systems and Software, 172:110869.

Dalla Palma, S., Di Nucci, D., Palomba, F., and Tamburri, D. A. (2021). Within-project defect prediction of infrastructure-as-code using product and process metrics. IEEE Transactions on Software Engineering.

de Feijter, R., Overbeek, S., van Vliet, R., Jagroep, E., and Brinkkemper, S. (2018). Devops competences and maturity for software producing organizations. In Enterprise, Business-Process and Information Systems Modeling, pages 244-259. Springer.

Domingos, P. (2012). A few useful things to know about machine learning. Communications of the ACM, 55(10):78-87.

Eifert, T., Eisen, K., Maiwald, M., and Herwig, C. (2020). Current and future requirements to industrial analytical infrastructure-part 2: smart sensors. Analytical and bioanalytical chemistry, 412(9):2037-2045.

Forsgren, N. and Kersten, M. (2018). Devops metrics. Communications of the ACM, 61(4):44-48.

Fursin, G. (2021). Collective knowledge: organizing research projects as a database of reusable components and portable workflows with common interfaces. Philosophical Transactions of the Royal Society A, 379(2197):20200211.

García, Á. L., De Lucas, J. M., Antonacci, M., Zu Castell, W., David, M., Hardt, M., Iglesias, L. L., Moltó, G., Plociennik, M., Tran, V., et al. (2020). A cloud-based framework for machine learning workloads and applications. IEEE access, 8:1868118692. 
Granlund, T., Stirbu, V., and Mikkonen, T. (2021). Towards regulatory-compliant mlops: Orazivio's journey from a machine learning experiment to a deployed certified medical product. SN Computer Science, 2(5):1-14.

Jabbari, R., bin Ali, N., Petersen, K., and Tanveer, B. (2016). What is devops? a systematic mapping study on definitions and practices. In Proceedings of the Scientific Workshop Proceedings of XP2016, pages 1-11.

Karamitsos, I., Albarhami, S., and Apostolopoulos, C. (2020). Applying devops practices of continuous automation for machine learning. Information, 11(7):363.

Karn, R. R., Kudva, P., and Elfadel, I. A. M. (2019). Dynamic autoselection and autotuning of machine learning models for cloud network analytics. IEEE Transactions on Parallel and Distributed Systems, 30(5):1052-1064.

Kitchenham, B., Brereton, O. P., Budgen, D., Turner, M., Bailey, J., and Linkman, S. (2009). Systematic literature reviews in software engineering-a systematic literature review. Information and software technology, 51(1):7-15.

Kitchenham, B. A. and Charters, S. (2007). Guidelines for performing systematic literature reviews in software engineering. Technical Report EBSE 2007-001, Keele University and Durham University Joint Report.

Langley, P. and Simon, H. A. (1995). Applications of machine learning and rule induction. Communications of the ACM, 38(11):54-64.

Leite, L., Rocha, C., Kon, F., Milojicic, D., and Meirelles, P. (2019). A survey of devops concepts and challenges. ACM Comput. Surv., 52(6).

Li, Y., Jiang, Z. M., Li, H., Hassan, A. E., He, C., Huang, R., Zeng, Z., Wang, M., and Chen, P. (2020). Predicting node failures in an ultra-large-scale cloud computing platform: an aiops solution. ACM Transactions on Software Engineering and Methodology (TOSEM), 29(2):1-24.

Liu, Y., Ling, Z., Huo, B., Wang, B., Chen, T., and Mouine, E. (2020). Building a platform for machine learning operations from open source frameworks. IFAC-PapersOnLine, 53(5):704-709. 3rd IFAC Workshop on Cyber-Physical Human Systems CPHS 2020.

Lwakatare, L. E., Crnkovic, I., and Bosch, J. (2020). Devops for ai-challenges in development of ai-enabled applications. In 2020 International Conference on Software, Telecommunications and Computer Networks (SoftCOM), pages 1-6. IEEE.

Moher, D., Stewart, L., and Shekelle, P. (2015). All in the family: systematic reviews, rapid reviews, scoping reviews, realist reviews, and more. Systematic reviews, 4(1):12.

Oluyisola, O. E., Bhalla, S., Sgarbossa, F., and Strandhagen, J. O. (2021). Designing and developing smart production planning and control systems in the industry 4.0 era: a methodology and case study. Journal of Intelligent Manufacturing, pages 1-22.

Pääkkönen, P. and Pakkala, D. (2020). Extending reference architecture of big data systems towards machine learning in edge computing environments. Journal of Big Data, $7(1): 1-29$.

Rossel, S. (2017). Continuous Integration, Delivery, and Deployment: Reliable and faster software releases with automating builds, tests, and deployment. Packt Publishing Ltd. 
Saltz, J., Shamshurin, I., and Crowston, K. (2017). Comparing data science project management methodologies via a controlled experiment. In Hawaii International Conference on System Sciences 2017.

Schlossnagle, T. (2018). Monitoring in a devops world. Communications of the ACM, 61(3):58-61.

Sculley, D., Holt, G., Golovin, D., Davydov, E., Phillips, T., Ebner, D., Chaudhary, V., Young, M., Crespo, J.-F., and Dennison, D. (2015). Hidden technical debt in machine learning systems. Advances in neural information processing systems, 28:2503-2511.

Shahin, M., Babar, M. A., and Zhu, L. (2016). The intersection of continuous deployment and architecting process: practitioners' perspectives. In Proceedings of the 10th ACM/IEEE International Symposium on Empirical Software Engineering and Measurement, pages $1-10$.

Spjuth, O., Frid, J., and Hellander, A. (2021). The machine learning life cycle and the cloud: implications for drug discovery. Expert Opinion on Drug Discovery, pages 1-9.

Tom, E., Aurum, A., and Vidgen, R. (2013). An exploration of technical debt. Journal of Systems and Software, 86(6):1498-1516. 